Palavras chave:

Guanandi

Anatomia da madeira

Madeira juvenil

Fibras,

Vasos

Histórico:

Recebido 07/01/2014

Aceito 02/03/2016

Keywords:

Guanandi

Wood anatomy

Juvenile wood

Fibers

Vessels

Correspondência: simonesimoessi@gmail.com
Ricardo Marques Barreiros', Simone Simões Amaral', Carlos Alberto Oliveira de Matos', Alex Siqueira Costa'

\section{ESTUDO QUANTITATIVO DA VARIAÇÃO ESTRUTURAL MORFOLÓGICA NA MADEIRA DE Calophyllum brasiliense}

RESUMO: As características silviculturais e tecnológicas favoráveis da espécie Calophyllum brasiliense Camb. têm levado ao surgimento de plantios comerciais. De modo geral, a literatura apresenta somente dados médios e gerais sobre algumas variáveis desta espécie, não informando dados específicos a determinadas variáveis antecessoras ao momento do estudo tecnológico, como idade, espaçamento, entre outros, talvez porque se trata de uma espécie de floresta nativa. Dada a lacuna existente quanto à caracterização da madeira de guanandi, o objetivo deste trabalho foi estudar quantitativamente: (i) a variação estrutural morfológica das principais células do lenho, e (ii) a delimitação entre madeira juvenil e adulta, para a espécie proveniente de plantio puro, cujas variáveis antecessoras são conhecidas. Foram amostradas cinco espécimes, dentro de uma população de 135 árvores, com 13 anos de idade e espaçamento $4,0 \mathrm{~m} \times 2,5 \mathrm{~m}$. Os resultados indicaram valores médios de II34; 21,02; I2,88 e 4,07 $\mu \mathrm{m}$ para o comprimento, largura, diâmetro do lume e espessura média da parede das fibras, respectivamente. A madeira de guanandi com 13 anos de idade foi caracterizada como juvenil.

\section{QUANTITATIVE STUDY OF MORPHOLOGICAL STRUCTURAL VARIATION IN THE Calophyllum brasiliense WOOD}

ABSTRACT: Favorable silvicultural and technological characteristics of the species Calophyllum brasiliense Camb. have led to the emergence of commercial plantations. Scientific literature offers only medium and general data on some variables of this species, not reporting specific variables prior to the time of the study, such as age, spacing, among others, perhaps because the wood is mostly from native forests. Given the knowledge gap regarding the characterization of guanandi wood, the aim of this work was to quantitatively study: (i) the morphological structural variation of the wood cells, and (ii) the distinction between juvenile and mature wood, for the species from a pure plantation, whose growth history are known. Five specimens were sampled, within a population of I 35 trees, with 13 years of age and $4.0 \times 2.5 \mathrm{~m}$ spacing. The results indicated average values of 1134 , $21.02,12.88$ and $4.07 \mu \mathrm{m}$ for the length, width, and diameter of the lumen and mean wall fiber thickness, respectively. 13-years-old guanandi wood was characterized as juvenile. 


\section{INTRODUÇÃO}

Segundo Lisboa et al. (20 I 2), a espécie Calophyllum brasiliense Camb. (guanandi) é bastante recomendada para fins econômicos. Para Urzedo et al. (20l3), o guanandi pode ser uma alternativa à espécie Tectona grandis e outras mais, que são também recomendadas comercialmente. Além do mais, o guanandi possui o especial atributo de estar adaptado a vários sítios edafoclimáticos, desde o sul do Brasil até a América Central.

Dentre os usos comerciais da madeira de guanandi, Urzedo et al. (2013) citam a construção naval, a marcenaria, a carpintaria, etc. A casca e a folha desta espécie também têm sido utilizadas no desenvolvimento de fármacos para o combate de doenças degenerativas como a leishmaniose (AYALA et al., 20I3; BRENZAN et al., 20I2).

Em termos de plasticidade, Oliveira e Joly $(2012)$ e Souza et al. (2007) trataram a espécie $C$. brasiliense como tolerante a inundação, com preferência em colonizar solos com alta saturação hídrica, sendo considerada de grande plasticidade ecológica. Patreze et al. (20l2) destacaram que o $C$. brasiliense apresenta ocorrência natural generalizada e padrões geográficos de variação genética adaptativa.

Sob o aspecto de produção de mudas, Lisboa et al. (20/2), ao estudarem mudas de guanandi, perceberam baixa produção de massa seca da parte aérea e do sistema radicular, além de crescimento em diâmetro e altura praticamente linear. Segundo os autores, estes acontecimentos podem ter ocorrido devido à capacidade de adaptação da espécie a ambientes adversos.

Do ponto de vista silvicultural, Brenes e Montagnini (2006) percebeu que essa espécie apresenta melhor crescimento em povoamentos puros do que em povoamentos mistos. Ciriello (2009) detalha que aos 6 anos o primeiro desbaste pode ser realizado e aos 12 anos pode ser feito o segundo. Nesta segunda etapa, a madeira apresenta de 25 a 30 $\mathrm{cm}$ de DAP e $16 \mathrm{~m}$ de altura.

Apesar das excelentes características silviculturais, a madeira de guanandi é ainda pouco estudada, carecendo de novas pesquisas que venham caracterizála tecnologicamente. Para Patreze et al. (20I2), muito pouco se sabe sobre a madeira dessa espécie. Além do mais, Sette Júnior et al. (20I2) apontam que o rápido crescimento e o aumento da produtividade das florestas plantadas podem acarretar alterações na qualidade do lenho, sendo fundamental a sua avaliação. Palermo et al. (2013) reforçaram a importância de se conhecer a multiplicidade das propriedades de um material heterogêneo como a madeira, para seu uso correto e Oliveira et al. (2012) abordaram a caracterização anatômica, como ferramenta capaz de proporcionar esse conhecimento.

Durante o crescimento, as árvores produzem diferentes tipos de tecido lenhoso, conforme descrito por Palermo et al. (20/3). Segundo os autores, o xilema produzido nos primeiros anos até certa idade cambial é denominado lenho juvenil e apresenta propriedades físicas, mecânicas, químicas e anatômicas diferentes do xilema produzido após certa idade cambial, ou seja, do lenho adulto. Gatto et al. (2007) argumentaram que a proporção de madeira juvenil no mercado é crescente, sendo importante definir a idade aproximada em que ocorre a transição da madeira juvenil para madeira adulta.

Salvo as descrições anatômicas gerais realizadas por Mainieri e Chimelo (1989), Muñiz et al. (2012) e Richter e Dallwitz (2000), não foram encontradas informações a respeito da anatomia do lenho da espécie $C$. brasiliense.

Assim, objetivou-se com este trabalho estudar quantitativamente a variação estrutural das principais células do lenho de guanandi, como também a sua delimitação entre a madeira juvenil e adulta.

\section{MATERIAL E MÉTODOS}

\section{Coleta e preparação do material}

Para este estudo, foram utilizadas árvores de Calophyllum brasiliense Camb., com 13 anos de idade, conduzidas numa área de $1350 \mathrm{~m}^{2}$, em espaçamento 4,0 m x 2,5 m, no município de Adrianópolis, estado do Paraná, Brasil.

De uma população de 135 árvores, com classes diametrais variando de 4 a $20 \mathrm{~cm}$ de Diâmetro à Altura do Peito (DAP), foram sorteadas e amostradas 5 árvores representativas para as classes diametrais entre 10 e 20 $\mathrm{cm}$. Dessas árvores retiraram-se discos de $2,5 \mathrm{~cm}$ de espessura a $0 \%, 25 \%, 50 \%, 75 \%$ e $100 \%$ da altura comercial. Os discos foram descascados e cortados em 4 cunhas de $90^{\circ}$, passando pela medula. Uma dessas cunhas foi utilizada para a análise anatômica microscópica (maceração e lâminas histológicas). As análises foram realizadas no Laboratório de Anatomia da Madeira, do Câmpus de Itapeva da Universidade Estadual Paulista "Júlio de Mesquita Filho". A preparação das cunhas, a partir dos discos, foi nas condições seca ao ar, com aproximadamente $12 \%$ de umidade. 


\section{Confecção de lâminas histológicas e determinação das dimensões dos vasos}

Para o preparo e montagem das lâminas histológicas, optou-se por trabalhar apenas com o disco a $25 \%$ da altura comercial, por este pertencer à porção da árvore mais utilizada comercialmente, além do que, foi a altura que ainda continha algum cerne, pois na altura de $50 \%$ já não foi detectada sua presença. Foram obtidos corpos de prova padronizados, apresentando cerca de I,5 $\mathrm{cm}^{2}$ de área no plano transversal, em formato piramidal e orientados na transição cerne/alburno, que é a região mais indicada para os estudos anatômicos. As lâminas de madeira para a montagem de lâminas histológicas semipermanentes, conforme Johansen (1940) e Sass (195I), foram seccionadas em micrótomo Leica com I $8 \mu \mathrm{m}$ de espessura nos planos transversal (onde foram medidos os diâmetros dos vasos), longitudinal radial e longitudinal tangencial. Na seção transversal, foi coletada imagem digital do lenho através de microscópio óptico (Leica - DM 2500) acoplado à câmara Leica DFC-295 para a determinação dos diâmetros tangenciais dos vasos e de seus lumes (Figura la), bem como da frequência dos vasos (Figura Ib).

\section{Maceração e determinação das dimensões das fibras}

A partir das cunhas retiradas a $0 \%, 25 \%$ e $50 \%$ da altura comercial, foram obtidas baguetas de $3 \mathrm{~mm}$ de espessura a cada $\mathrm{Icm}$ do raio, no sentido medulaalburno (Figura 2).
As baguetas foram picadas em pequenas lascas e dissociadas através do método de Franklin, conforme Johansen (1940) e Sass (1951). Imagens das dimensões das fibras foram coletadas utilizando microscópio óptico acoplado à câmara digital. Foram feitas medições do comprimento (Figura 3a), largura e diâmetro do lume (Figuras 3b), e calculada a espessura média da parede da fibra, que é a metade da diferença entre a largura da fibra e o diâmetro do lume.

\section{Análises estatísticas}

Para todos os elementos medidos, determinaram-se medidas de tendência central (média) e de dispersão (desvio padrão), bem como o intervalo de confiança. $\bigcirc$ número mínimo necessário de amostras dos elementos anatômicos mensuradas, para garantir um erro amostral máximo de 10\%, em relação à média, foi conforme sugerido por Calonego et al. (2005) e descrito nas equações I e 2. Em que n: é o número de repetições; t: estatística " $t$ " de Student; $\mathrm{s}^{2}$ : variância amostral; E: erro admissível; m: média dos valores amostrados.

$N=\left(t^{2} \cdot s^{2}\right) \cdot E^{-2}$

$E^{2}=(0,1 \cdot m)^{2}$

Para a identificação do ponto que delimita madeira juvenil e adulta utilizou-se a análise de regressão com nível de significância de $5 \%(\alpha=0,05)$.

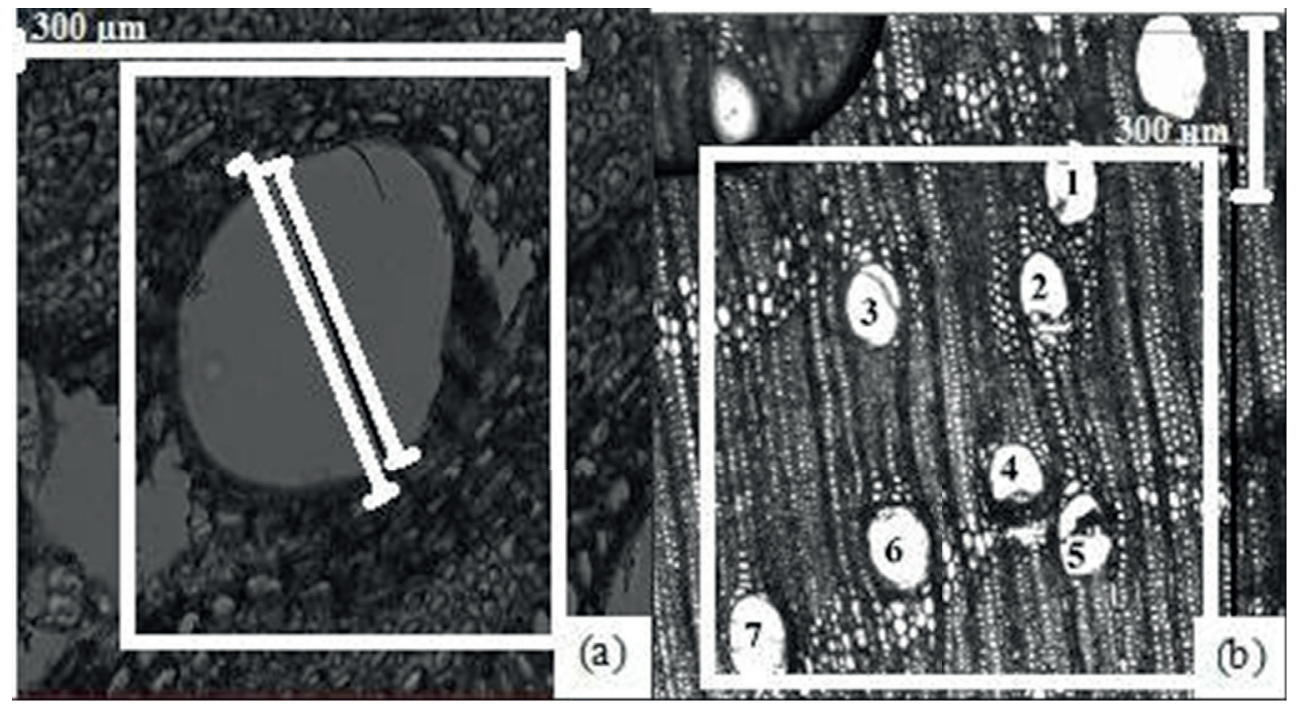

FIGURA 1 Seção transversal do lenho: (a) largura do vaso e diâmetro do lume - 200x; (b) frequência dos vasos por mm² - 20x. Barra de escala: $300 \mu \mathrm{m}$.

FIGURE 1 Cross section of wood: (a) vessel width and lumen diameter - 200x; (b) vessels frequency per mm² - 20x. Scale bar: 300 $\mu \mathrm{m}$. 

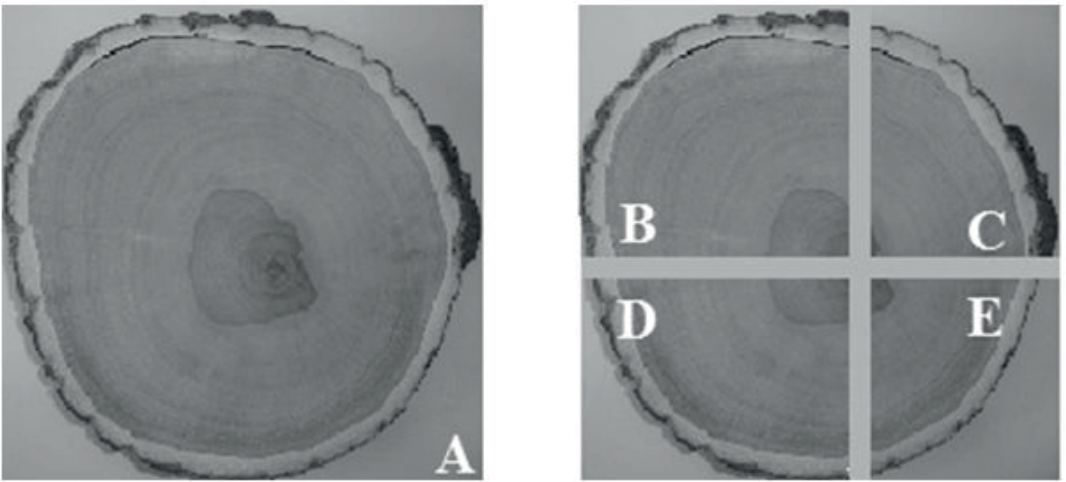

FIGURA 2 Esquema de preparação das amostras para a maceração.

FIGURE 2 Scheme from preparing samples for the maceration.

\section{RESULTADOS E DISCUSSÃO}

\section{Quantificação dos elementos estruturais do lenho}

$\mathrm{Na}$ Tabela I, foram dispostos os valores da estatística descritiva para as dimensões das fibras (comprimento, largura total, diâmetro do lume e espessura da parede) e dos elementos de vasos (diâmetro do lume e frequência).

A frequência dos elementos de vaso na madeira de guanandi, coletados nos cinco espécimes, apresentaram um valor médio de 7,42 vasos $\cdot \mathrm{mm}^{-2}$, com valores variando entre 5 e 10 vasos $\cdot \mathrm{mm}^{-2}$. $O$ diâmetro médio do lume dos vasos foi de I I4,40 $\mu \mathrm{m}$, mas variou de $8 \mathrm{I}, 70$ a I 5 I,70 $\mu \mathrm{m}$. A literatura reporta valores próximos para a mesma espécie de madeira. Muñiz et al. (20I2) encontraram a frequência média de 5,47 vasos. $\mathrm{mm}^{-2}$ e valor médio do diâmetro dos poros de 155,05 $\mu \mathrm{m}$. Para esta espécie, Mainieri e Chimelo ( 1989) obtiveram valores de frequência e diâmetro de vasos entre 2 e 7 vasos $\cdot \mathrm{mm}^{-2}$ e 120 e $200 \mu \mathrm{m}$, respectivamente. Richter e Dallwitz (2000) encontraram uma frequência de 4 a 10 vasos $\cdot \mathrm{mm}^{-2}$ e comprimento médio dos elementos vasculares de $670 \mu \mathrm{m}$, variando de 280 a I I $70 \mu \mathrm{m}$.

O comprimento médio das fibras foi de II 34,00 $\mu \mathrm{m}$, com valores variando entre $682,90 \mu \mathrm{m}$ e 1557,00 $\mu \mathrm{m}$. A espessura média da parede foi de $4,07 \mu \mathrm{m}$ e variou de 2,35 a 6,00 $\mu \mathrm{m}$. O diâmetro do lume variou de $7,03 \mu \mathrm{m}$ a $21,96 \mu \mathrm{m}$, com valor médio de 12,88 $\mu \mathrm{m}$. A largura média da fibra foi de $21,02 \mu \mathrm{m}$, variando de 14,06 $\mu \mathrm{m}$ a 31,04 $\mu \mathrm{m}$. Richter e Dallwitz (2000) verificaram valores mínimo, médio e máximo de 780, 1280 e 1670 $\mu \mathrm{m}$, respectivamente, para o comprimento das fibras.
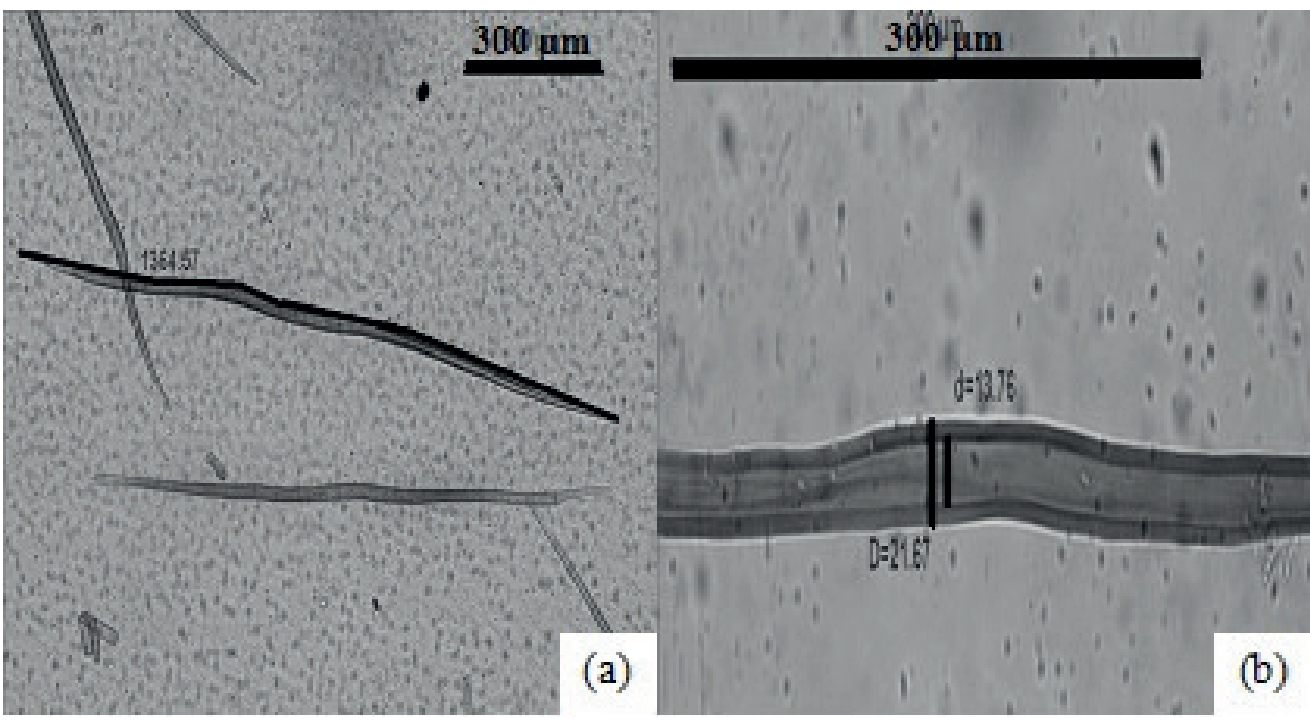

FIGURA 3 Determinação das fibras do lenho: (a) comprimento - 50x; (b) largura da fibra e diâmetro do lume - 200x. Barra de escala: $300 \mu \mathrm{m}$.

FIGURE 3 Determination of wood fibers: (a) length - 50x; (b) fiber width and lumem diameter - 200x. Scale bar: $300 \mu \mathrm{m}$. 
TABELA 1 Estatística descritiva das dimensões dos elementos que compõe o lenho.

TABLE 1 Descriptive statistics of dimensions of the elements that make up the wood.

\begin{tabular}{ccccccccc}
\hline $\begin{array}{c}\text { Estruturas } \\
\text { anatômicas }\end{array}$ & & Mín. & Méd. & Máx. & S & C.V. & N & I.C \\
\hline \multirow{2}{*}{ Vasos } & Dp & 81,70 & 114,40 & 151,70 & 21,59 & 18,87 & 60,00 & {$[108,787: 119,941]$} \\
& F & 5,00 & 7,42 & 10,00 & 1,06 & 14,32 & 60,00 & {$[7.142: 7.691]$} \\
& C & 682,90 & 1134,00 & 1557,00 & 140,36 & 12,38 & 1248,00 & {$[1126,603: 1142,193]$} \\
\multirow{2}{*}{ Fibras } & Lf & 14,06 & 21,02 & 31,04 & 2,05 & 9,76 & 1248,00 & {$[20,903: 21,131]$} \\
& Df & 7,03 & 12,88 & 21,96 & 1,90 & 14,76 & 1248,00 & {$[12,771: 12,983]$} \\
& E & 2,35 & 4,07 & 6,00 & 0,59 & 14,53 & 1248,00 & {$[4,037: 4,103]$} \\
\hline
\end{tabular}

Mín, Méd, Máx: valor mínimo, médio e máximo das variáveis em $(\mu \mathrm{m})$; S: desvio padrão; $n$ : unidades amostrais; I.C: intervalo de confiança; Dp: diâmetro do lúme do poro $(\mu \mathrm{m})$; F: frequência (poros/mm2); C: comprimento da fibra $(\mu \mathrm{m})$; Lf: largura total da fibra $(\mu \mathrm{m})$; Df: diâmetro do lúme da fibra ( $\mu \mathrm{m})$; E: espessura da parede da fibra $(\mu \mathrm{m})$.

\section{Delimitação entre madeira juvenil e adulta}

Gatto et al. (2007) e Palermo et al. (2013) destacaram que o comprimento das fibras é o principal indicador do ponto de transição de madeira juvenil para adulta. Desta forma, para delimitação entre madeira juvenil e adulta, utilizou-se de regressão linear ( $\alpha=5 \%)$, considerando o comprimento das fibras em relação à distância radial medula/casca.

$\mathrm{Na}$ Tabela 2, tem-se a equação da reta que descreve a existência da relação linear, além dos valores do coeficiente de determinação $\left(R^{2}\right)$.

Os resultados estatísticos evidenciaram a existência de relação linear positiva, entre o comprimento das fibras e a distância radial medula/ casca. A relação linear foi altamente significativa (Valor de $P<5 \%)$. No entanto, os valores dos coeficientes de determinação variaram de baixos a moderados, explicando somente $17,72 \%$ da variação observada no conjunto dos cinco espécimes.

$\mathrm{Na}$ Figura 4 foram plotados os valores do comprimento das fibras ao longo da altura comercial em

TABELA 2 Relação entre o comprimento das fibras e a distância radial medula/casca, para os espécimes representantes das cinco classes diametrais.

TABLE 2 Relationship between the fibers length and the radial distance medulla/bark, to the representatives of the diametric classes five specimens.

\begin{tabular}{ccccc}
\hline Árvores & Classe & $\begin{array}{c}\text { Modelo linear } \\
\text { simples ajustado }\end{array}$ & $\begin{array}{r}\mathrm{R}^{2} \\
(\%)\end{array}$ & Valor de P \\
\hline A & $10 \vdash 12$ & $\mathrm{y}=23,64 . \mathrm{a}+1126,35$ & 6,76 & 0,0004 \\
$\mathrm{~B}$ & $12 \vdash 14$ & $\mathrm{y}=33,51 . \mathrm{a}+957,33$ & 21,01 & $<0,0001$ \\
C & $14 \vdash 16$ & $\mathrm{y}=29,69 . \mathrm{a}+1021,08$ & 38,44 & $<0,0001$ \\
D & $16 \vdash 18$ & $\mathrm{y}=24,40 . \mathrm{a}+957,10$ & 32,49 & $<0,0001$ \\
E & $18 \vdash 20$ & $\mathrm{y}=39,89 . \mathrm{a}+1009,93$ & 40,96 & $<0,0001$ \\
A-E & $10 \vdash 20$ & $\mathrm{y}=24,41 . \mathrm{a}+1038,42$ & $17,72<0,0001$ \\
\hline
\end{tabular}

função da distância no sentido medula/casaca, para cada classe diametral.

Observa-se o aumento praticamente linear no comprimento das fibras no sentido medula/casca, o que, segundo Palermo et al. (20l3), é característico de lenho juvenil, cujo comprimento das fibras apresentou dimensões em contínuo desenvolvimento, não havendo um ponto de delimitação entre madeira juvenil e adulta.

Gatto et al. (2007), ao estudarem a madeira de plátano, observaram que até o décimo quarto ano de vida desta espécie ocorreu um aumento no comprimento da fibra, seguida de estabilização e diminuição a partir do vigésimo ano. Os autores perceberam que a idade de segregação encontrada na madeira de plátano, para o comprimento das fibras, foi de 14 anos.

\section{CONCLUSÕES}

A partir dos resultados obtidos e apresentados para a madeira de Calophyllum brasiliense, concluiuse que: $O$ comprimento das fibras e outras variáveis relacionadas a este elemento anatômico aumentaram na direção radial do fuste, no sentido medula/casca. As árvores analisadas apresentaram variação radial dos elementos anatômicos do lenho. Com 13 anos de idade, a madeira foi caracterizada como juvenil, não havendo um ponto de delimitação entre madeira juvenil e adulta.

\section{AGRADECIMENTOS}

À Fundação de Amparo à Pesquisa do Estado de São Paulo (FAPESP) pelo financiamento do projeto e concessão de bolsas de Iniciação Científica e Treinamento Técnico. 

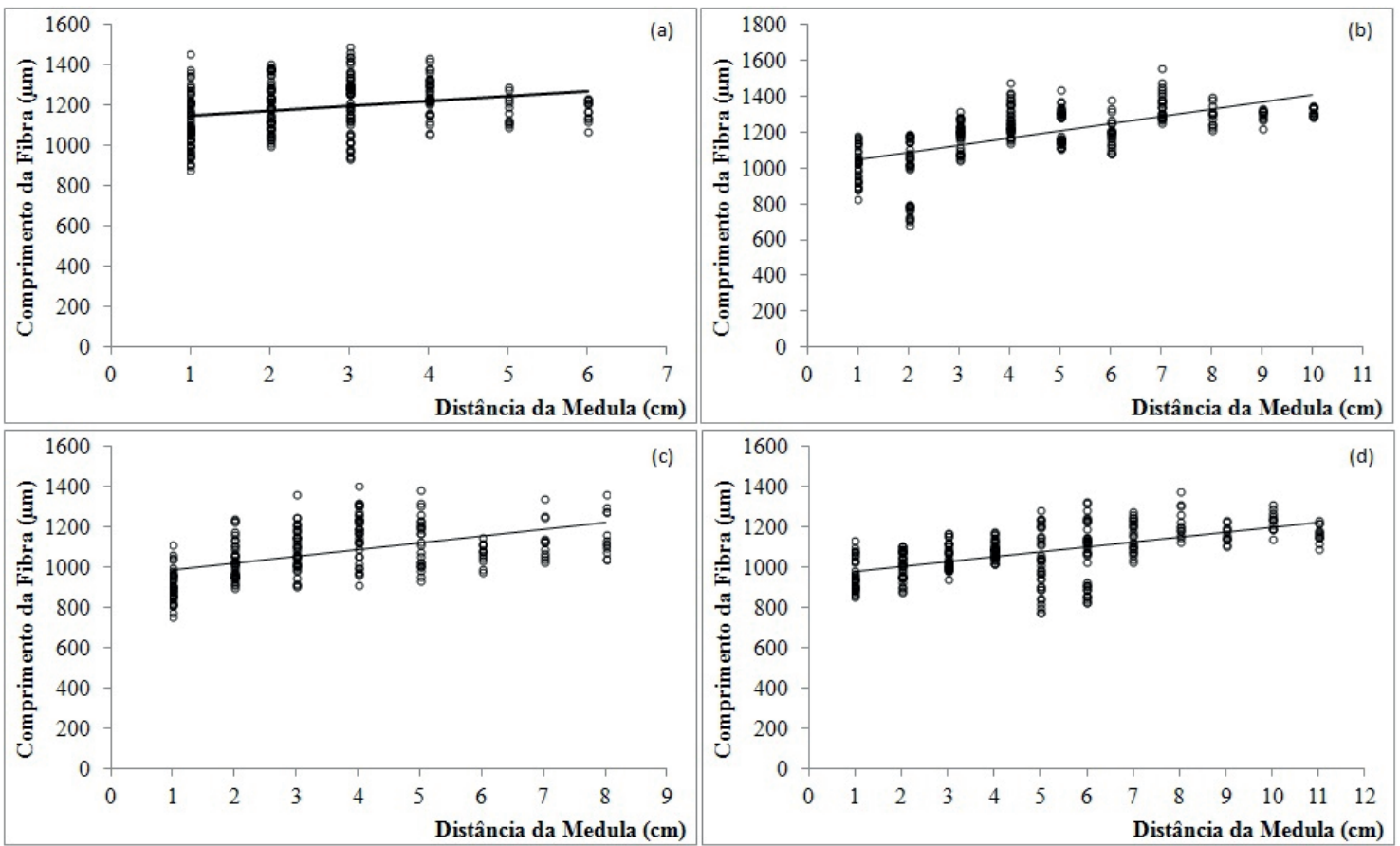

(d)

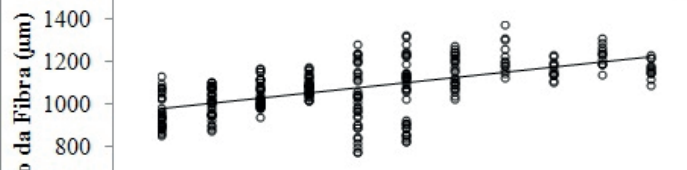

600

600
400

छ Distância da Medula (cm)
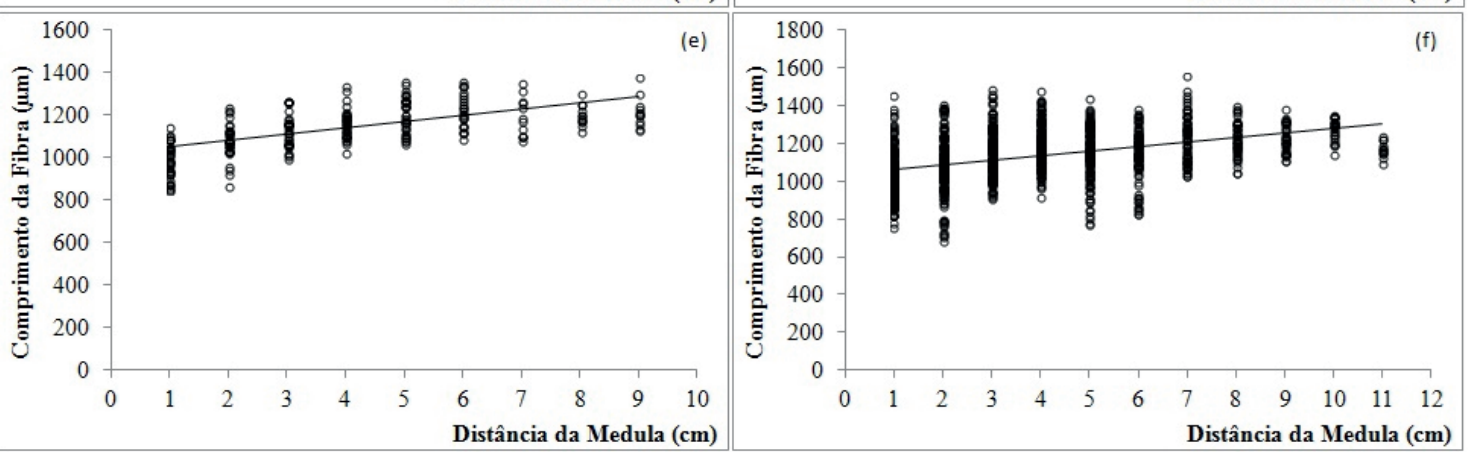

FIGURA 4 Variação do comprimento da fibra $(\mu \mathrm{m})$ com a distância no sentido medula/casca: (a) árvore A- diâmetro de 10 a $12 \mathrm{~cm}$; (b) árvore B- diâmetro de 12 a 14 cm; (c) árvore C- diâmetro de 14 a 16 cm; (d) árvore D- diâmetro de 16 a 18 cm; (e) árvore E- diâmetro de 18 a $20 \mathrm{~cm}$; (f) todas as árvores analisadas.

FIGURE 4 Variation of length fiber $(\mu \mathrm{m})$ with the distance in the direction medulla/bark: (a) tree A- diameter of 10 to $12 \mathrm{~cm}$; (b) tree B- diameter of 12 to $14 \mathrm{~cm}$; (c) tree C- diameter of 14 to $16 \mathrm{~cm}$; (d) tree D- diameter of 16 to $18 \mathrm{~cm}$; (e) tree E- diameter of 18 to $20 \mathrm{~cm}$; (f) all trees analyzed.

\section{REFERÊNCIAS}

AYALA, T. B.; HUITRÓN, R. L.; LÓPEZ, E. M. S.; CHILPA, R. R.; LÓPEZ, E. R.; PINEDA, B.; CAMPOS, O. N. M.; CHAPUL, L. S.; PINZÓN, E.; SOLIS, C. T.; ADAYA, D. S.; CHAVERRÍ, J. P.; RÍOS, C.; DE LA CRUZ, V. P.; RAMOS, M. T. Antioxidant properties of xanthones from Calophyllum brasiliense: prevention of oxidative damage induced by $\mathrm{FeSO}_{4}$. BMC Complementary and Alternative Medicine, v. 13, n. 262, p. I-15Oct. 2013.

BRENES, A. R.; MONTAGNINI, F. Growth, productivity, aboveground biomass, and carbon sequestration of pure and mixed native tree plantations in the Caribbean lowlands of Costa Rica. Forest Ecology and Management, v. 232, n. 1/3, p. 168-178, Aug. 2006.
BRENZAN, M. A.; SANTOS, A. O.; NAKAMURA, C. V.; DIAS FILHO, B. P.; UEDA-NAKAMURA, T.; YOUNG, M. C. M.; CÔRREA, A. G.; ALVIM JÚNIOR, J.; MORGADO-DÍAZ, J. A.; CORTEZ, D. A. G. Effects of (-) mammea $A / B B$ isolated from Calophyllum brasiliense leaves and derivatives on mitochondrial membrane of Leishmania amazonensis. Phytomedicine, v. 19, n. 3/4, p. 223-230, Feb. 2012.

CALONEGO, F. W.; SEVERO, E. T. D.; ASSI, P. P. Mensuração do comprimento das fibras para a determinação da madeira juvenil em Eucalyptus citriodora. Scientia Forestalis, n. 68, p. ||3-|2|, ago. 2005.

CIRIELLO, P. Caracterização anatômica macroscópica das madeiras utilizadas para laminação na região de CuritibaPR. Referência, São Paulo, v. I I, n. 94, p. 39-45, ago. 2009. 
GATTO, D. A.; HASELEIN, C. R.; BULIGON, E. A.; CALEGARI, L.; STANGERLIN, D. M.; OLIVEIRA, L. da S. Estimativa da idade de segregação do lenho juvenil e adulto para Platanus x acerifolia (Ait.) Willd. Cerne, Lavras, v. 13, n. 4, p. 393398, out./dez. 2007.

JOHANSEN, D. A. Plant microtechnique. New York: MacGraw-Hill, 1940. 533 p.

LISBOA, A. C.; SANTOS, P.S.; OLIVEIRANETO, S. N. de; CASTRO, D. N. de; ABREU, A. H. M. Efeito do volume de tubetes na produção de mudas de Calophyllum brasiliense e Toona ciliata. Revista Árvore, Viçosa, v. 36, n. 4, p. 603-609, 2012.

MAINIERI, C.; CHIMELO, J. P. Fichas de características das madeiras brasileiras. São Paulo: IPT, 1989. 4 I8 p.

MUÑIZ, G. I. B.; NISGOSKI, S.; SHARDOSIN, F. Z.; FRANÇA, R. F. Anatomia do carvão de espécies florestais. Cerne, Lavras, v. 18, n. 3, p. 47I-477, jul./set. 2012.

OLIVEIRA, J. G. L. de; OLIVEIRA, J. T. da S.; ABAD, J. I. M.; SILVA, A. G. da; FIELDLER, N. C.; VIDAURE, G. B. Parâmetros quantitativos da anatomia da madeira de eucalipto que cresceu em diferentes locais. Revista Árvore, Viçosa, v. 36, n. 3, p. 559-567, 2012.

OLIVEIRA, V. C. de; JOLY, C. A. Tolerância ao alagamento de Calophyllum brasiliense Camb.: respostas morfológicas, fisiológicas e de crescimento: (Clusiaceae). Trees, v. 24, p. I85-193, 2010.

PALERMO, G. P. de M.; LATORRACA, J. V. de F; SEVERO, E. T. D.; NASCIMENTO, A. M. do; REZENDE, M. A. Delimitação entre os lenhos juvenil e adulto de Pinus elliottii Engelm. Revista Árvore, Viçosa, v. 37, n. I, p. 191-200, 2013.
PATREZE, C. M.; FELIX, D. B.; SCARANO, F. R.; ALVESFERREIRA, M. Isolating of a putative glyceraldehyde-3 phosphate dehydrogenase (GAPDH) from Calophyllum brasiliense, an important tropical forest tree. Silvae Genetica, v. 6I, n. I/2, p. 44-5I, 2012.

RICHTER, H. G.; DALLWITZ, M. J. Commercial timbers: descriptions, illustrations, identification, and information retrieval. 2000. Disponível em: <http:// delta-intkey.com/wood/pt/www/gutcabra.htm $>$. Acesso em: 14 fev. 2014.

SASS, J. E. Botanical microtechnique. lowa: State College Press, 195I. 228 p.

SETTE JÚNIOR, C. R.; OLIVERA, I. R. de; TOMAZELLO FILHO, M.; YAMAJI, F. M.; LACLAU, J. P. Efeito da idade e posição de amostragem na densidade e características anatômicas da madeira de Eucalyptus grandis. Revista Árvore, Viçosa, v. 36, n. 6, p. II83-I 190, 2012.

SOUZA, A. M. de; CARVALHO, D. de; VIEIRA, F. de A.; NASCIMENTO, L. H. do; LIMA, D. C. Estrutura genética e espacial de populações naturais de Calophyllum brasiliense Camb. em mata de galeria. Cerne, Lavras, v. 13, n. 3, p. 239-247, jul./set. 2007.

URZEDO, D. I. de; FRANCO, M. P.; PITOMBO, L. M.; CARMO, J. B. do. Effects of organic and inorganic fertilizers on greenhouse gas (GHG) emissions in tropical forestry. Forest Ecology and Management, v. 310, n. 15, p. 3744, Dec. 2013. 
